\title{
Multiple antibiotic susceptibility of polyphosphate kinase mutants (ppk1 and ppk2) from Pseudomonas aeruginosa PAO1 as revealed by global phenotypic analysis
}

\author{
Javiera Ortiz-Severín", Macarena Varas', Catalina Bravo-Toncio', Nicolás Guiliani and Francisco P Chávez ${ }^{1 *}$
}

\begin{abstract}
Background: Pseudomonas aeruginosa is known to be a multidrug resistant opportunistic pathogen. Particularly, P. aeruginosa PAO1 polyphosphate kinase mutant (ppk1) is deficient in motility, quorum sensing, biofilm formation and virulence.

Findings: By using Phenotypic Microarrays (PM) we analyzed near 2000 phenotypes of $P$. aeruginosa PAO1 polyP kinase mutants ( $p p k 1$ and $p p k 2$ ). We found that both $p p k$ mutants shared most of the phenotypic changes and interestingly many of them related to susceptibility toward numerous and different type of antibiotics such as Ciprofloxacin, Chloramphenicol and Rifampicin.

Conclusions: Combining the fact that ppk1 mutants have reduced virulence and are more susceptible to antibiotics, polyP synthesis and particularly PPK1, is a good target for the design of molecules with anti-virulence and anti-persistence properties.
\end{abstract}

Keywords: Antibiotic susceptibility, Polyphosphate kinase, Pseudomonas, Phenome, Phenotypic microarrays, Multidrug resistance

\section{Findings}

Pseudomonas aeruginosa is a major opportunistic pathogen frequently involved in hospital-acquired infections and can produce severe pneumonia, burn wound infections, and sepsis. Particularly, multidrug resistant (MDR) variants are emerging rapidly in the clinic for this pathogen. In addition, $P$. aeruginosa resistance rates have increased to available antimicrobial agents, limiting the choice of available anti-infective chemicals [1]. Looking for alternatives with economic and human health impact, new antimicrobial agents with novel biological targets or strategies are desperately needed to combat highly resistant $P$. aeruginosa infections [2,3]. Current original strategies combine the reduction of bacterial

\footnotetext{
* Correspondence: fpchavez@uchile.cl

'Systems Microbiology Laboratory, Department of Biology, Faculty of Science, University of Chile, Las Palmeras 3425, Nuñoa, Santiago, Chile Full list of author information is available at the end of the article
}

virulence with a simultaneous increase of animal host defence, instead of eradicating the pathogen $[4,5]$.

Inorganic polyphosphate (polyP) are essential for bacterial resilience during stress and stringencies, cellular motility, biofilm formation and virulence [6]. Many bacterial pathogens knockout of polyP synthesis gene ( $p p k 1)$ result in cellular defects, particularly in the context of virulence toward the host they invade [6,7]. Specifically, a Pseudomonas aeruginosa PAO1 mutant of polyP synthesis (Pappk1) was impaired in motility, biofilm development, quorum sensing and virulence in ocular and burned-mouse models $[8,9]$. Moreover, this mutant exhibited reduced viability after exposure to a $\beta$-lactam antibiotic [10]. Similar results were reported in $p p k 1$ mutants from S. typhimurium and S. dublin that used Polymyxin B15 antibiotic [11]. In addition to homologues of PPK1, another widely conserved polyP enzyme is PPK2, which, in contrast to the ATP-dependent polyP synthetic activity of PPK1, preferentially catalyses the 
reverse reaction, polyP-driven synthesis of GTP from GDP [12]. The fact that polyP is involved in bacterial virulence and resilience processes makes it an attractive target for antimicrobial agents [2].

To provide a more complete analysis of $P$. aeruginosa polyP synthesis mutants ( $p p k 1$ and $p p k 2)$ phenotypes and to obtain greater insight into the physiological changes and particularly chemical susceptibility we used Phenotype Microarray (PM) technology [13]. Biolog Phenotype MicroArrays studies in Pseudomonas aeruginosa PAO1 have been used to facilitate further characterization of known mutation strains and for testing bioinformatic predictions for mutations in hypothetical or unknown genes [14]. We used $P$. aeruginosa polyphosphate synthesis knockout mutants $p p k 1$ (PA5242) and ppk2 (PA0141) from a $P$. aeruginosa Mini- Tn5-Tcr gene knockout mutants collection [15]. We confirmed by PCR both mutations and bacterial cell suspensions from all strains were inoculated into each of the 20 PM plates for full metabolic profiling according to standard protocols recommended by Biolog Inc. for Pseudomonas strains [14,16]. The PM plates were located in an aerobic OmniLog incubator reader set at $30^{\circ} \mathrm{C}$ which collected data every 15 min over a 72-h period. PM tests were conducted in duplicate, and the plates were also examined visually at the end of each incubation period for independent confirmation. The OmniLog ${ }^{\ominus}$ V. 1.5 comparison module and the average height parameter were used for data analysis with standard thresholds for detection. A consensus graphical profiles for all metabolic and sensitivity tests for each mutant were generated using two independent runs (Additional file 1: Figure S1).

For visualization and clustering data analysis, Multiexperiment Viewer (MeV version 4.6) software was used. $\mathrm{MeV}$ is part of the TM4 Microarray Software Suite, an open source system for statistical and clustering analysis of omics data [17].

Comparing the metabolic and sensitivity capabilities between mutants and their isogenic parent strains we show that both mutant strains behave similar in nutrient utilization (PM1-10) and sensitivity to chemicals (PM1120) assays (Figure 1, Table 1). This indicates that these two strains shared most of the phenotypic patterns and differed only in a few features (Figure 1, Additional file 2: Tables S1-S4). Particularly interesting is the altered isoleucine metabolism found exclusively in the $p p k 1 \mathrm{mu}-$ tant (Additional file 2: Table S2). In Pseudomonas persistence of $p p k 1$ mutant in the stationary phase was significantly affected $[18,19]$. In contrast, the main metabolic pathways were not significantly influenced by the loss of $p p k 1$ as revealed from respiration patterns of the ppk mutants in phenotypic microarrays. Our results are in agreement with those reported in Pseudomonas putida KT2440 where accumulation of inorganic polyphosphate

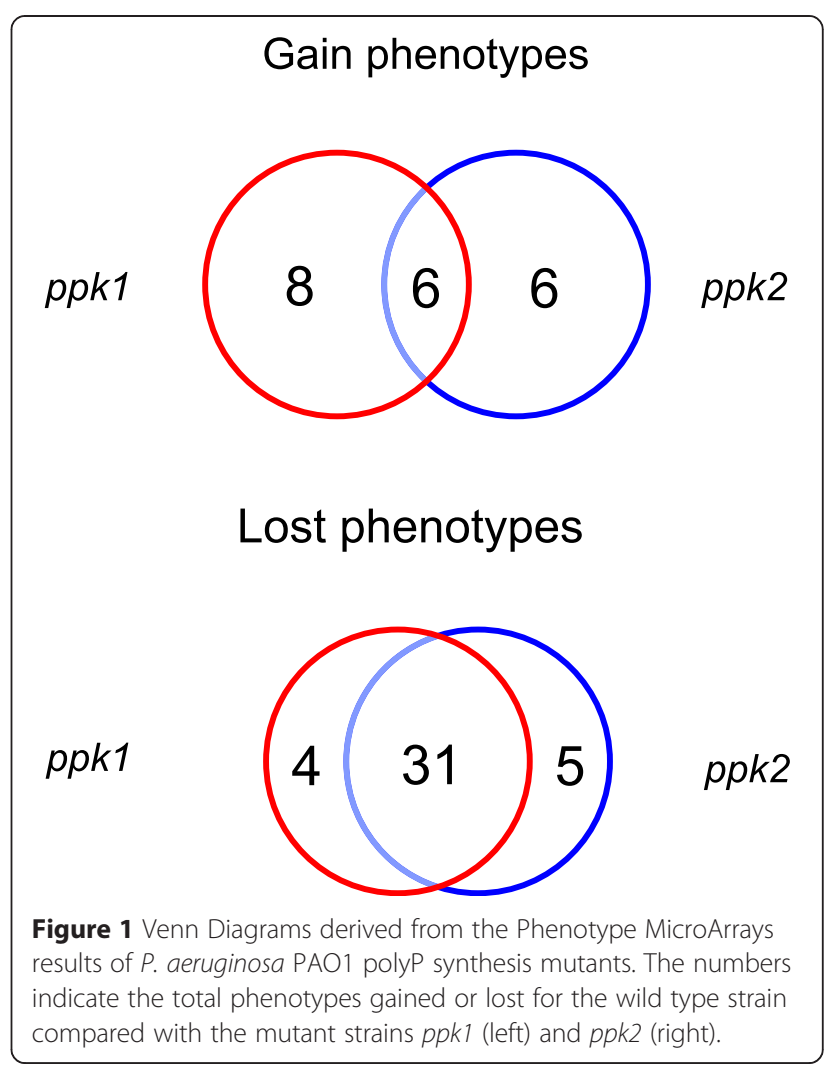

enables stress endurance and catalytic vigour but the major metabolic routes were not significantly influenced by the loss of ppk1 [18].

The respiration patterns of $P$. aeruginosa PAO1 $p p k 1$ and $p p k 2$ associated with the metabolism of carbon (PM1-PM2), nitrogen (PM3), phosphorous and sulfur (PM4) sources were relatively moderate as compared to previous unpublished PM results from our laboratory with $\Delta p p k 1$ mutant from Escherichia coli (Additional file 1: Figures S2 and S3). We speculate that the apparent metabolic robustness in Pseudomonas ppk1 mutant is due to in the $E$. coli genome there is only one gene orthologous to the PPK1 protein [12]. In contrast, in Pseudomonas aeruginosa PAO1 the presence of another ortholog (PPK2) can compensate the lost of $p p k 1$ gene, in fact, despite the absence of detectable PPK1 activity ( $<1 \%$ of wild type), $\Delta p p k 1$ mutants still possess as much as $20 \%$ of polyP of the wild type levels [12].

Our results show that the majority of phenotypic changes observed were coincident in $p p k 1$ and $p p k 2$ mutants (Figure 1, Table 1). We found only few differences among phenotypes between both ppk mutants (Additional file 2: Tables S1-S4). We were able to identify novel phenotypes for polyP synthesis mutants in $P$. aeruginosa PAO1 and found that both mutants are susceptible to multiple antibiotics (Figure 2). 
Table 1 Summary of $P$. aeruginosa PAO1 polyP synthesis mutants (ppk1 and ppk2) common gain and lost phenotypes

\begin{tabular}{|c|c|c|c|}
\hline Test & ppk1 difference & ppk2 difference & Mode of action \\
\hline & Gain phenotype & Gain phenotype & \\
\hline L-Arginine & 50 & 75 & C-Source, carboxylic acid \\
\hline His-Met & 61 & 56 & N-Source, peptide \\
\hline Carbenicillin & 158 & 71 & Wall, lactam \\
\hline Cefsulodin & 80 & 80 & Wall, cephalosporin \\
\hline Ruthenium red & 78 & 110 & Respiration, mitochondrial $\mathrm{Ca}^{2+}$ porter \\
\hline \multirow[t]{2}{*}{ Erythromycin } & 67 & 80 & Protein synthesis, 505 ribosomal subunit, macrolide \\
\hline & Lost phenotype & Lost phenotype & \\
\hline D-Gluconic acid & -112 & -106 & C-Source, carboxylic acid \\
\hline D-Mannitol & -68 & -57 & C-Source, carbohydrate \\
\hline L-Valine & -60 & -58 & N-Source, amino acid \\
\hline L-Methionine Sulfone & -69 & -95 & S-Source, organic \\
\hline Orphenadrine & -78 & -146 & Anti-cholinergic \\
\hline Patulin & -534 & -495 & Microtubulin polymerization inhibitor, antifungal \\
\hline 2,2'-Dipyridyl & -401 & -397 & Chelator, lipophilic \\
\hline Sodium Arsenite & -332 & -242 & Toxic anion \\
\hline Sodium Arsenate & -305 & -321 & Toxic anion, P04 analog \\
\hline Dichlofluanid & -271 & -119 & Fungicide, phenylsulphamide \\
\hline Thiamphenicol & -269 & -193 & Protein synthesis, amphenicol \\
\hline Chloramphenicol & -209 & -173 & Protein synthesis, amphenicol \\
\hline Chlorodinitrobenzene & -182 & -187 & Oxidizes sulfhydryls, depletes glutathione \\
\hline 8-Hydroxyquinoline & -181 & -230 & Chelator, lipophilic \\
\hline Nafcillin & -163 & -172 & Wall, lactam \\
\hline Antimony (III) chloride & -158 & -153 & Toxic cation \\
\hline 1,10-Phenanthroline monohydrate & -152 & -171 & Chelator, lipophilic \\
\hline Captan & -152 & -113 & Fungicide, carbamate \\
\hline Sulfathiazole & -148 & -172 & Folate antagonist, PABA analog \\
\hline Gallic acid & -141 & -115 & Respiration, ionophore, $\mathrm{H}^{+}$ \\
\hline Poly-L-lysine & -140 & -63 & Membrane, detergent, cationic \\
\hline Protamine sulfate & -129 & -109 & Membrane, nonspecific binding \\
\hline Sulfadiazine & -127 & -107 & Folate antagonist, PABA analog \\
\hline Oxacillin & -124 & -130 & Wall, lactam \\
\hline Myricetin & -113 & -122 & DNA \& RNA synthesis, polymerase inhibitor \\
\hline Nickel chloride & -113 & -80 & Toxic cation \\
\hline Plumbagin & -106 & -118 & Oxidizing agent \\
\hline Oxophenylarsine & -99 & -161 & Tyrosine phosphatase inhibitor \\
\hline trans-Cinnamic acid & -98 & -90 & Respiration, ionophore, $\mathrm{H}^{+}$ \\
\hline EDTA & -67 & -88 & Chelator, hydrophilic \\
\hline Glycine hydroxamate & -66 & -136 & tRNA synthetase \\
\hline
\end{tabular}

Interestingly, we observed susceptibility to various antibiotic families with different mechanisms of action such as Penicillins (Nafcillin, Oxacillin), Ansamycins (Streptomycin), Sulfonamides (Sulfadiazine, Sulfathiazole) and Others (Chloramphenicol, Thiamphenicol). To confirm this finding, we used two additional wellestablished methods for antimicrobial susceptibility testing. The results from the M.I.C.Evaluator ${ }^{\text {TM }}$ (Oxoid) and Etest $^{\oplus}$ (Biomeriux) corroborated the susceptibility of $p p k$ mutants observed in the PM results (Additional file 2: 


\section{Chemical sensitivity (PM11 to PM20)}

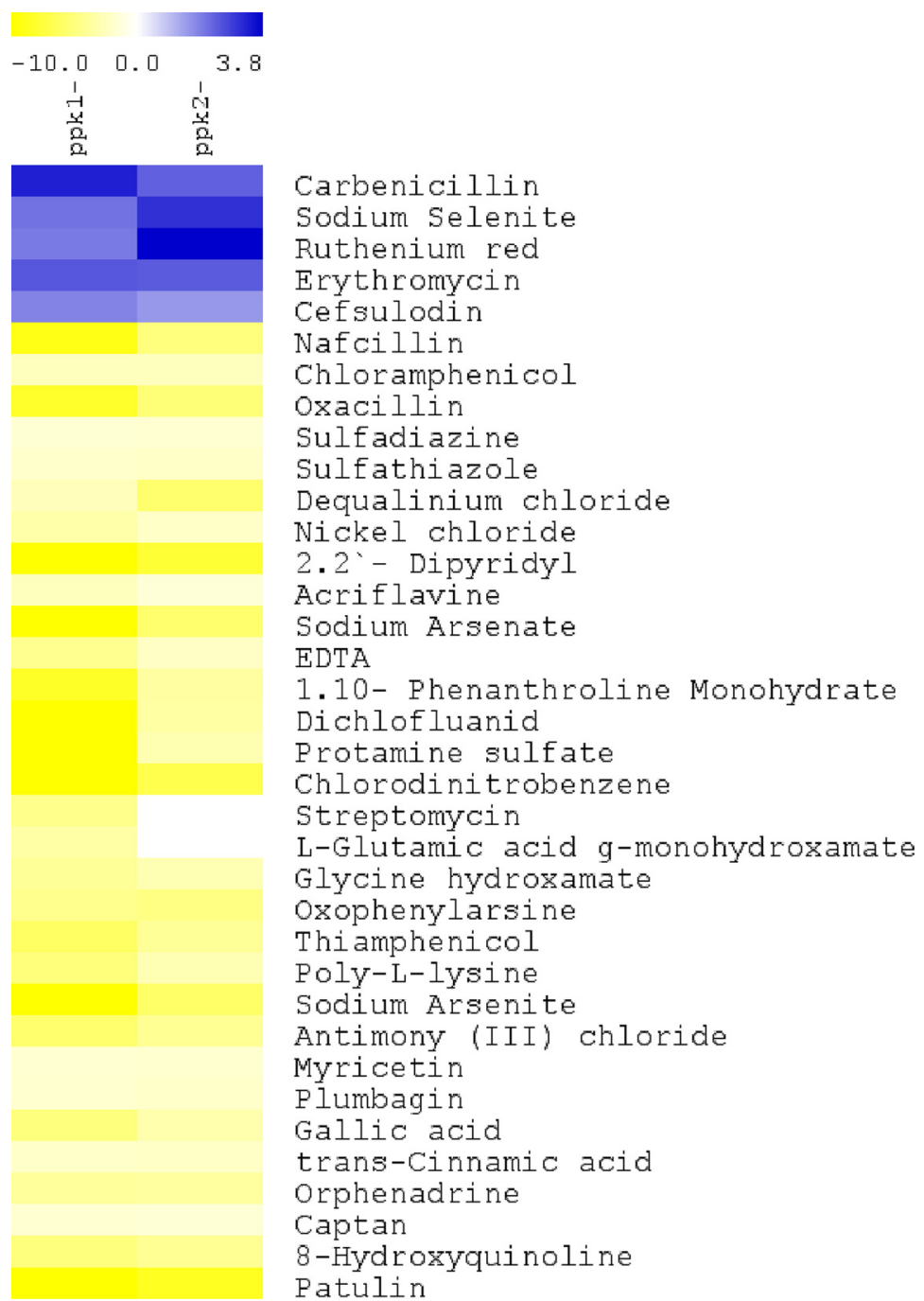

Figure 2 PolyP kinases (ppk1 and ppk2) mutants from P. aeruginosa PAO1 are susceptible to multiple antibiotics. Clustering analysis of chemical sensitivity tests (PM11-20) from P. aeruginosa PAO1 ppk1 and ppk2 mutants. Gain (blue) and lost (yellow) phenotypes were standardized by dividing the respiration values of the mutants by the respective values of the wild type strain, for each phenotype.

Table S5). The minimal inhibitory concentration (MIC) parameters for both $p p k$ mutants were particularly interesting for the antibiotics Rifampicin, Imipinen and Ciprofloxacin, either because Pseudomonas is intrinsically resistant or because recent strains highly resistant to these antibiotics have been found recently [20]. It should be noted that concentrations of antibiotics used in the PM experiments are set according to the minimal inhibitory concentration for $E$. coli. It is possible that other antibiotic susceptibilities have not been detected since both the control strain and the mutants were resistant.

Taking into account that various bacterial regulatory genes that participate in complex regulatory networks have been reported to influence both virulence and antibiotic resistance [20], polyP levels could affect regulators that control both antibiotic resistance and virulence.

Finally, our results support the recent finding that bacterial persistence, a phenomenon in which isogenic populations of antibiotic-sensitive bacteria produce rare cells that transiently become multidrug tolerant, is affected in ppk1 deficient strain [21]. This small fraction of cells that grow slowly explains bacterial antibiotic tolerance because the cellular targets disturbed by lethal antibiotics are much less vulnerable in slow-growing than in fast-growing cells. This is important because the presence of persister cells has been suggested to be one of 
the main reasons for failures in the treatment of these chronic diseases. Indeed, clinical isolates of Pseudomonas aeruginosa from cystic fibrosis patients show an increase in high persistence mutants the longer that these isolates remain in the host. This indicates that persistence plays a major role in the failure to remove these bacterial populations from the cystic fibrosis lung [20].

Consequently, polyP synthesis, and particularly PPK1, in bacterial pathogens exhibits a potential target for antimicrobial drug design because combines the reduction of bacterial virulence and persistence, while simultaneously increasing susceptibility to antibiotics as we describe here by using PM technology. This could positively impact the host health for clearing the bacterial infection. Considering that no PPK1 homologs have been identified in higherorder eukaryotes [22], our phenotypic sensibility results highlight the importance of polyP synthesis as a putative target for the treatment of this opportunistic bacterium.

\section{Additional files}

Additional file 1: Figure S1. Consensus graphical profile of metabolic and sensitivity tests of $P$. aeruginosa ppk1 (A) and ppk2 (B) mutants. Significant changes are enclosed in boxes. Yellow indicates that respiration rate of the wild type and mutant strains were similar. Red indicates faster respiration rate of the wild type (lost phenotype). Green indicates faster respiration rate of the mutant (gain phenotype). The quantitative difference values are shown in Table 1 and Supplementary tables. Figure S2. Clustering analysis of metabolic tests (PM1-PM8) and $\mathrm{pH}$ response (PM10) from P. aeruginosa PAO1 ppk1 and ppk2 mutants. Gain (blue) and lost (yellow) phenotypes were standardized by dividing the respiration value of the mutants by the value of the wild type strain for each phenotype. The results are shown separately for the different categories: carborn $(C)$ sources, nitrogen $(\mathrm{N})$ sources, phosphorus $(\mathrm{P})$ and sulfur sources (S), peptide nitrogen ( $\mathrm{N}$ ) sources and pH response.

Figure S3. Venn diagrams of phenotypic microarrays results from polyP synthesis mutants from E. coli K12 and P. aeruginosa PAO1. The numbers indicate the total phenotypes gained (A) or lost (B) between $P$. aeruginosa ppk1 and ppk2 mutants and E. coli $\Delta p p k 1$ mutant. Phenotypic microarray results from $E$. coli $\Delta p p k 1$ mutant were performed in a previous work (Unpublished results).

Additional file 2: Table S1. Summary of $P$. aeruginosa $p p k 1$ mutant gain and lost phenotypes. Table S2. Summary of $P$. aeruginosa ppk2 mutant gain and lost phenotypes. Table $\mathbf{S 3}$. Summary of unique $P$. aeruginosa ppk1 mutant gain and lost phenotypes. Table S4. Summary of unique $P$. aeruginosa ppk2 mutant gain and lost phenotypes. Table S5. Summary of antimicrobial susceptibility testing (M.I.C.Evaluator ${ }^{\mathrm{TM}}$ (Oxoid) and Etest ${ }^{\oplus}$ (Biomeriux) for P. aeruginosa ppk1 and ppk2 mutant strains.

\section{Abbreviations}

PPK: Polyphosphate kinase; PolyP: Polyphosphate; MDR: Multidrug resistance; PM: Phenotype Microarray (Biolog); PCR: Polymerase chain reaction; MIC: Minimal inhibitory concentration; Pappk1: Polyphosphate kinase 1 from Pseudomonas aeruginosa PAO1.

\section{Competing interests}

The authors declare that they have no competing interests.

\section{Authors' contributions}

All authors contributed extensively to the work presented in this paper. JOS, MV and CBT performed all the experiments. JOS and MV interpreted the PM data and conducted all bioinformatics analyses. FCH jointly conceived the study with NG and wrote the paper. All authors discussed the results and implications and commented on the manuscript at all stages. All authors read and approved the final manuscript.

\section{Acknowledgements}

We gratefully acknowledge Dr. Michael Ziman (Biolog, Inc.) for all the support in the phenotypic microarray experiments. We thank Nicole Molina and members of SysmicroLab for helpful technical assistance. This work was supported by a Fondecyt Grant (1120209) from Conicyt. JOS, MV and CBT are Conicyt fellows for postgraduate studies.

\section{Author details}

'Systems Microbiology Laboratory, Department of Biology, Faculty of Science, University of Chile, Las Palmeras 3425, Nuñoa, Santiago, Chile. ${ }^{2}$ Bacterial Communication Laboratory, Department of Biology, Faculty of Science, University of Chile, Las Palmeras 3425, Ñuñoa, Santiago, Chile.

Received: 2 February 2015 Accepted: 8 April 2015

Published online: 25 April 2015

\section{References}

1. Tam VH, Rogers CA, Chang K-T, Weston JS, Caeiro J-P, Garey KW. Impact of multidrug-resistant Pseudomonas aeruginosa bacteremia on patient outcomes. Antimicrob Agents Chemother. 2010;54:3717-22.

2. Chávez FP, Lagos CF, Reyes-parada M, Guiliani N, Jerez CA. Polyphosphate Synthesis as a Target for Novel Antibiotics. Curr Enzym Inhib. 2011;7:163-8.

3. Sintim HO, Smith JA, Wang J, Nakayama S, Yan L. Paradigm shift in discovering next-generation anti-infective agents: targeting quorum sensing, c-di-GMP signaling and biofilm formation in bacteria with small molecules. Future Med Chem. 2010;2:1005-35.

4. Kaplan F, Badri DV, Zachariah C, Ajredini R, Sandoval FJ, Roje S, et al. Bacterial attraction and quorum sensing inhibition in Caenorhabditis elegans exudates. J Chem Ecol. 2009;35:878-92.

5. Swem LR, Swem DL, O'Loughlin CT, Gatmaitan R, Zhao B, Ulrich SM, et al. A quorum-sensing antagonist targets both membrane-bound and cytoplasmic receptors and controls bacterial pathogenicity. Mol Cell. 2009;35:143-53.

6. Brown MRW, Kornberg A. The long and short of it - polyphosphate, PPK and bacterial survival. Trends Biochem Sci. 2008;33:284-90.

7. Brown MRW, Kornberg A. Inorganic polyphosphate in the origin and survival of species. Proc Natl Acad Sci U S A. 2004;101:16085-7.

8. Rashid MH, Rumbaugh K, Passador L, Davies DG, Hamood AN, Iglewski BH, et al. Polyphosphate kinase is essential for biofilm development, quorum sensing, and virulence of Pseudomonas aeruginosa. Proc Natl Acad Sci U S A. 2000;97:9636-41.

9. Rashid MH, Kornberg A. Inorganic polyphosphate is needed for swimming swarming, and twitching motilities of Pseudomonas aeruginosa. Proc Natl Acad Sci U S A. 2000;97:4885-90.

10. Fraley CD, Rashid MH, Lee SSK, Gottschalk R, Harrison J, Wood PJ, et al. A polyphosphate kinase 1 (ppk1) mutant of Pseudomonas aeruginosa exhibits multiple ultrastructural and functional defects. Proc Natl Acad Sci U S A. 2007;104:3526-31.

11. Kim K-S, Rao NN, Fraley CD, Kornberg A. Inorganic polyphosphate is essential for long-term survival and virulence factors in Shigella and Salmonella spp. Proc Natl Acad Sci U S A. 2002;99:7675-80.

12. Zhang $H$, Ishige K, Kornberg A. A polyphosphate kinase (PPK2) widely conserved in bacteria. Proc Natl Acad Sci U S A. 2002;99:16678-83.

13. Bochner BR. Global phenotypic characterization of bacteria. FEMS Microbiol Rev. 2009:33:191-205.

14. Johnson DA, Tetu SG, Phillippy K, Chen J, Ren Q, Paulsen IT. HighThroughput Phenotypic Characterization of Pseudomonas aeruginosa Membrane Transport Genes. PLoS Genet. 2008;4:11.

15. Jacobs MA, Alwood A, Thaipisuttikul I, Spencer D, Haugen E, Ernst S, et al. Comprehensive transposon mutant library of Pseudomonas aeruginosa. Proc Natl Acad Sci U S A. 2003;100:14339-44.

16. Wei Q, Tarighi S, Dötsch A, Häussler S, Müsken M, Wright VJ, et al. Phenotypic and Genome-Wide Analysis of an Antibiotic-Resistant Small Colony Variant (SCV) of Pseudomonas aeruginosa. PLoS One. 2011;6:e29276

17. Dudoit S, Gentleman RC, Quackenbush J. Open source software for the analysis of microarray data. Biotechniques. 2003;34:S45-51.

18. Nikel PI, Chavarría M, Martínez-García E, Taylor AC, de Lorenzo V. Accumulation of inorganic polyphosphate enables stress endurance and catalytic vigour in Pseudomonas putida KT2440. Microb Cell Fact. 2013;12:50. 
19. Kim HY, Schlictman D, Shankar S, Xie Z, Chakrabarty AM, Kornberg A. Alginate, inorganic polyphosphate, GTP and ppGpp synthesis co-regulated in Pseudomonas aeruginosa: Implications for stationary phase survival and synthesis of RNA/DNA precursors. Mol Microbiol. 1998;27:717-25.

20. Breidenstein EBM, de la Fuente-Núñez C, Hancock REW. Pseudomonas aeruginosa: all roads lead to resistance. Trends Microbiol. 2011;19:419-26.

21. Maisonneuve E, Castro-Camargo M, Gerdes K. (p)ppGpp controls bacterial persistence by stochastic induction of toxin-antitoxin activity. Cell. 2013;154:1140-50.

22. Hooley P, Whitehead MP, Brown MRW. Eukaryote polyphosphate kinases: is the "Kornberg" complex ubiquitous? Trends Biochem Sci. 2008;33:577-82.

\section{Submit your next manuscript to BioMed Central and take full advantage of:}

- Convenient online submission

- Thorough peer review

- No space constraints or color figure charges

- Immediate publication on acceptance

- Inclusion in PubMed, CAS, Scopus and Google Scholar

- Research which is freely available for redistribution 